\title{
Resonant Noise-Canceling Current Front-End for High-Resolution Impedance Sensing
}

\author{
G. Azzellino \\ Research Laboratory of Electronics \\ Massachusetts Institute of Technology \\ Cambridge (MA), USA \\ mozart87@mit.edu
}

\author{
A. Ragni, M. Carminati, G. Ferrari \\ Dipartimento di Elettronica, Informazione e Bioingegneria \\ Politecnico di Milano \\ Milano, Italy \\ giorgio.ferrari@polimi.it
}

\begin{abstract}
The ultimate resolution limit of the current frontend of wideband impedance detection circuits is set by the input total capacitance and input equivalent noise. We present a solution to reduce the noise of more than an order of magnitude when the capacitance cannot be further minimized. By introducing a properly-chosen inductor (and a proper and stable biasing network) it is possible to cancel the capacitive reactance at the resonance frequency $(\sim 10 \mathrm{MHz})$ which becomes the sensing frequency, thus significantly reducing the detection noise, while preserving the accuracy of the transfer function. A detailed analysis of the scheme, and its experimental validation with different inductors are presented. In all cases an improvement larger than 10 is achieved with an input-referred current spectral density of $\sim 1 \mathrm{pA} / \sqrt{\mathrm{Hz}}$ at the operating frequency of $\sim 20 \mathrm{MHz}$
\end{abstract}

Keywords-impedance sensors; lock-in, transimpedance amplifier, resonance, noise.

\section{INTRODUCTION}

The measurement of impedance is a very well consolidated sensing technology. Today, novel applications are emerging in different fields, enabled by the advancements of microfabrication technologies [1]. With the downscaling of the dimensions of devices and electrodes, the current signal flowing in the sensor impedance tends to become smaller and smaller, making high resolution (i.e. low noise) a pivotal aspect of modern impedance detection systems. The readout circuit must be often co-designed with the transducer, in order to push the performance limits, by custom-tailoring the electronics to the specific sensor. Impedance spectroscopy is often used to characterize novel materials and devices [2,3], while the final sensor implementation is based on tracking the impedance value over time at a single fixed frequency (or a few discrete tones). This is the case of capacitive sensors [4,5] as well as of AC-coupled conductance measurements, such as impedance cell counting in microfluidic systems [6] or hitless light monitoring in semiconductor waveguides [7]. Although in some cases, when the relative signal variation to be detected is in the order of $1 \mathrm{ppm}$, the instrument back-end (in particular digital lock-in amplifiers suffering from multiplicative flicker noise of the $\mathrm{A} / \mathrm{D}$ and $\mathrm{D} / \mathrm{A}$ chains [8]) might dictate the achievable resolution, in the majority of cases the noise of the front-end limits the instrument performance.
In the majority of applications mentioned above, the impedance measuring instrument is composed by a voltageforcing section and by a current-reading one, followed by a digital processor to calculate the impedance. Current sensing provides the following advantages with respect to other configurations (such as bridges or current-forcing schemes): (i) precise control of the voltage applied to the sensor, (ii) immunity to parasitics to ground. In fact, the sensor current is typically converted into a voltage by a transimpedance amplifier (TIA, Fig. 1a) which collects the current at the virtual ground and forces it in the feedback branch $\left(R_{F} \| C_{F}\right)$. Negative feedback grants: (i) very low input impedance, (ii) neutralization of any stray impedance from the input node to ground (such as $C_{i n}$ in Fig. 1a). Although not affecting the signal transfer function, the total input capacitance $C_{\text {in }}$ affects the stability of the feedback loop and the noise performance of the TIA. In fact, the input-equivalent voltage noise of the first operational amplifier is differentiated across $C_{\text {in }}$ producing a current noise increasing with frequency. Commonly, many efforts are devoted to the minimization of this capacitance, for instance by reducing the distance between the sensor and the integrated TIA $[1,9]$ or by choosing the right substrate for the fabrication of the sensor [10]. However, in some cases, this is not possible and the physical properties of the sensor (or its distance) set a large $C_{i n}$ (much larger than a few $\mathrm{pF}$ ) which corresponds, in combination with an input voltage noise which can be hardly reduced below $\sim 1 \mathrm{nV} / \sqrt{\mathrm{Hz}}$, to a large noise floor. This is especially detrimental for high-speed (i.e. largebandwidth) applications, such as the detection of ionic current through nanopores modulated by the passage of single macromolecules [9,11].

In this work we address the ultimate resolution performance of the TIA current front-end by proposing a simple and radical solution: adding an inductor at the input in order to lower the noise at the LC resonance frequency. We demonstrate a tenfold noise performance improvement with respect to the stateof-the-art current amplifiers [12,13]. The suppression of the equivalent voltage noise is achieved by nullifying the admittance at the virtual ground node with an inductive reactance tuned to counterbalance the capacitive one. This is perfectly suited for tracking the sensor impedance at a fixed sensing frequency, which is chosen as the resonance frequency of the LC tank. 


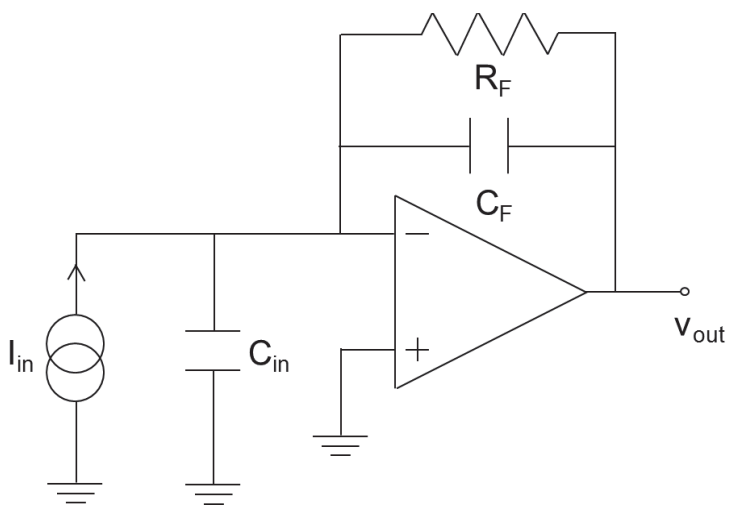

(a)

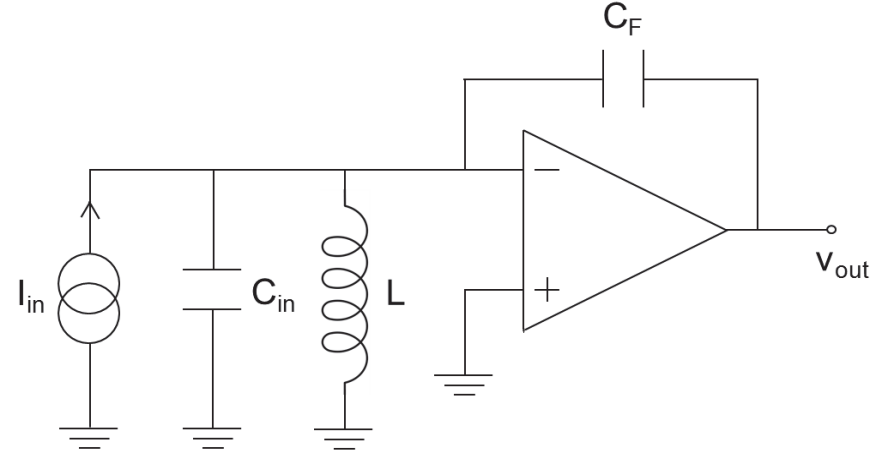

(b)

Fig. 1. (a) scheme of a traditional TIA. (b) scheme of the improved TIA modified with the inductor L at the virtual ground node of the current amplifier.

Beyond impedance, this solution is promising also for other applications where high-resolution detection of a modulated current is required, as in Raman spectroscopy. It is a noncontact analytical technique, for the analysis of molecules and organic compounds with growing applications in materials science and biomedicine. A laser beam interacts with molecular vibrations and the result of this interaction is typically acquired by a photodiode connected to TIA in a lockin scheme [14]. At the modulation frequency, the electronic noise of the amplifier is mainly due to the capacitance of the photodiode. If it becomes comparable with the laser shot noise, it may be a limitation for the current detection performance [15].

The main advantage of this technique is that the measurement signal-to-noise ratio (SNR) is increased by reducing the denominator (the noise) without influencing the numerator (the signal). In fact, the accuracy of the signal transfer function remains unaltered, differently from other resonant solutions where resonance is used to "amplify" the signal (numerator) at the price of reduced absolute accuracy $[16,17,18]$ or an increased complexity of the system to detect a phase shift [19] or a frequency shift [20]. Furthermore, any amplitude of input DC current can be managed by this scheme. The only critical aspects are: (i) the sensing frequency is single and depends on the value of $C_{i n}$, (ii) the losses of real inductors limit the improvements of this approach.

\section{ANALYSIS OF THE Proposed SOLUTION}

Figure 1a shows the scheme of a standard transimpedance amplifier where $C_{i n}$ is the sum of all capacitances related to the virtual ground node of the amplifier (capacitance of the sensor and the stray capacitances). The input current $i_{\text {in }}$ is converted into a voltage through the feedback impedance $R_{F}$ and $C_{F}$. The equivalent input noise is given by:

$$
\overline{i_{e q, T I A}^{2}} \cong \overline{i_{n}^{2}}+\frac{4 k T}{R_{F}}+\frac{\overline{e_{n}^{2}}}{R_{F}^{2}}+\overline{e_{n}^{2}}(2 \pi f)^{2}\left(C_{i n}+C_{F}\right)^{2}
$$

where $\overline{i_{n}^{2}}, \overline{e_{n}^{2}}$ are the equivalent input current noise and the equivalent input voltage noise, respectively, of the operational amplifier and $\mathrm{k}$ is the Boltzmann constant. The first three terms can be reduced using a FET operational amplifier and a large feedback resistor [12]. In order to extend the bandwidth of the TIA, advanced transimpedance architectures use the amplifier as an integrator stage [13]. The last term increases with the frequency becoming the limiting factor in many practical conditions. It is given by the voltage fluctuations of the virtual ground that produce a noise current through the impedance connected to the input of the amplifier, i.e. the input capacitance and the feedback capacitor at high frequency.

In order to reduce this noise, we have added an inductor at the input node of the amplifier as shown in Fig. 1b. At the resonant frequency given by $f_{\text {res }}=1 / 2 \pi \sqrt{L\left(C_{i n}+C_{F}\right)}$ the inductor and the capacitors are ideally an open circuit, hampering the transfer to the output of the equivalent voltage noise of the amplifier with a beneficial effect on the noise. Ideally the inductor is able to suppress completely the transfer of the voltage noise of the amplifier. The inductor's loss limits the reduction factor of the noise for two reasons. Firstly, the quality factor of the resonance $(Q)$ is a finite number and consequently the reactive elements are not an open circuit. It can be found that the noise term given by the voltage noise of the operational amplifier is reduced by $Q^{2}$ at the resonant frequency. Secondly, the losses of the inductor are a source of noise that we have modelled with a resistor in parallel to the inductor. At the resonant frequency $f_{\text {res }}$, the value of the resistor is related to the quality factor by the relation $R_{\text {loss }}=2 \pi f_{\text {res }} Q L$. The noise contribution due to the inductor losses can be estimated with the thermal noise of $R_{\text {loss }}$ :

$$
\overline{i_{\text {loss }}^{2}}\left(f_{\text {res }}\right) \cong \frac{4 k T}{R_{\text {loss }}}=\frac{4 k T}{2 \pi f_{\text {res }} Q L}=4 k T \frac{1}{Q} \sqrt{\frac{C_{\text {in }}+C_{F}}{L}}
$$

The equivalent input noise of the proposed circuit at the resonant frequency is therefore: 


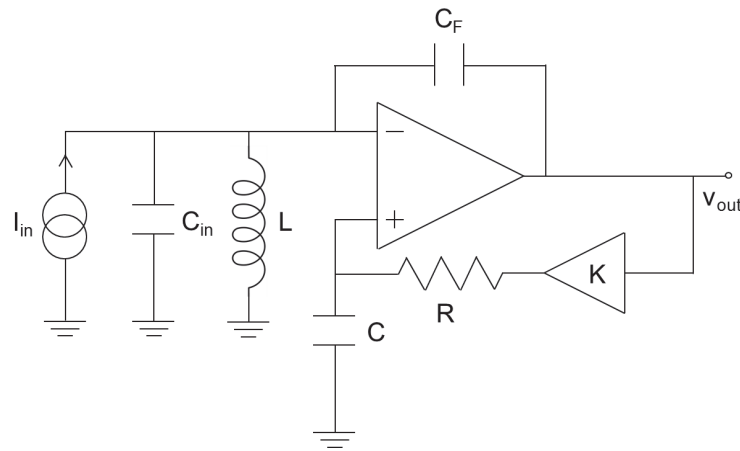

Fig. 2. Scheme of the additional network needed to set the TIA DC bias.

$$
\overline{i_{\text {eq,res }}^{2}}\left(f_{\text {res }}\right) \cong \overline{e_{n}^{2}}(2 \pi f)^{2}\left(\frac{C_{\text {in }}+C_{F}}{Q}\right)^{2}+4 k T \frac{1}{Q} \sqrt{\frac{C_{\text {in }}+C_{F}}{L}}
$$

As experimentally demonstrated in the section IV, the noise of proposed solution can be more than one order of magnitude lower than the noise of a standard transimpedance amplifier in the same operating conditions.

It is important to highlight that the noise suppression operated by the inductor does not affect the accuracy of the frequency response of the circuit at the resonant frequency. Indeed, the loop gain is

$$
G_{\text {loop }}(s)=-\frac{s^{2} L C_{F}}{1+s L / R_{\text {loss }}+s^{2} L\left(C_{\text {in }}+C_{F}\right)} A(s)
$$

where $A(s)$ is the gain of the operational amplifier. At the resonant frequency the magnitude of the loop gain is enhanced by a factor $Q$ with respect to the value of a standard transimpedance amplifier, thus granting an excellent virtual ground. Therefore, the input current signal $i_{\text {in }}$ entirely flows through the feedback capacitor fixing the gain of the amplifier to $v_{\text {out }} / i_{\text {in }}=1 / s C_{F}$ irrespective of the inductor value and of the quality factor of the resonance Being the gain of the resonant amplifier equal to the gain of an integrator stage operated at the same frequency, the reduction of the noise provided by the inductor directly increases the signal-to-noise ratio of the current (or impedance) measurement.

Finally, we note that the inductor provides a lowimpedance path to ground for the DC current at the input node. Consequently, the proposed circuit can operate with any DC bias of the device under test. On the contrary, a standard transimpedance amplifier has a maximum DC input current limited by the saturation of the voltage amplifier. A large DC current requires a correspondently small value of the feedback resistor $R_{F}$ to prevent the saturation of the amplifier with a detrimental effect on the noise performance.

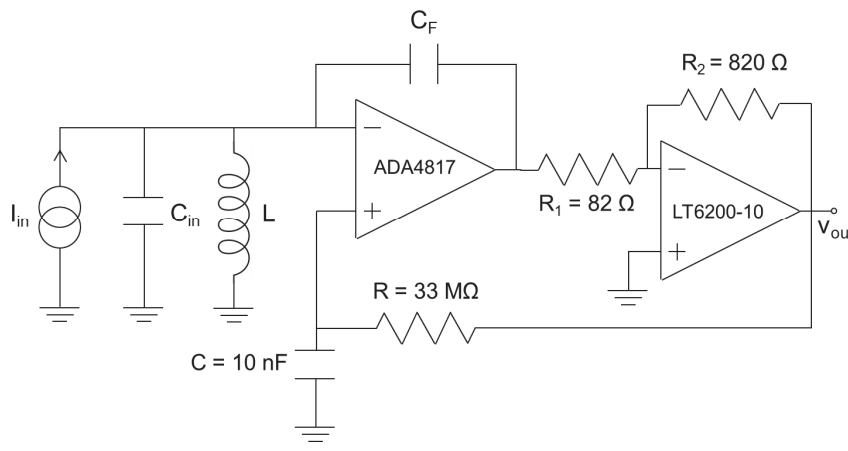

Fig. 3. Scheme of the circuit presented in this work, with the details of chosen off-the-shelf components.

\section{EXAMPLE OF IMPLEMENTATION}

The proposed circuit cannot be directly implemented in many practical cases. The pure capacitive feedback of the amplifier and the inductor at the input node set to zero the loop gain of the circuit at zero frequency, as shown in eq. (4). Being in open loop configuration, the voltage output of the operational amplifier would easily saturate to the maximum value. We address this issue by introducing an additional network that is able to fix the bias point of the operational amplifier at DC, as shown in Fig. 2. The additional network is composed by a gain stage $K$ and a low-pass filter. It sets the voltage of the noninverting input of the operational amplifier to keep the DC value of the output voltage around zero. At the resonant frequency the variation of the amplifier output does not change the voltage of the noninverting node, thanks to the strong attenuation of the low-pass filter. Thus, the additional network does not affect the frequency response of the amplifier at the frequency of the input signal. Assuming the path through the gain stage $\mathrm{K}$ and the low-pass filter actives at frequencies much lower than the resonant frequency, the loop gain of the additional DC loop can be approximated with

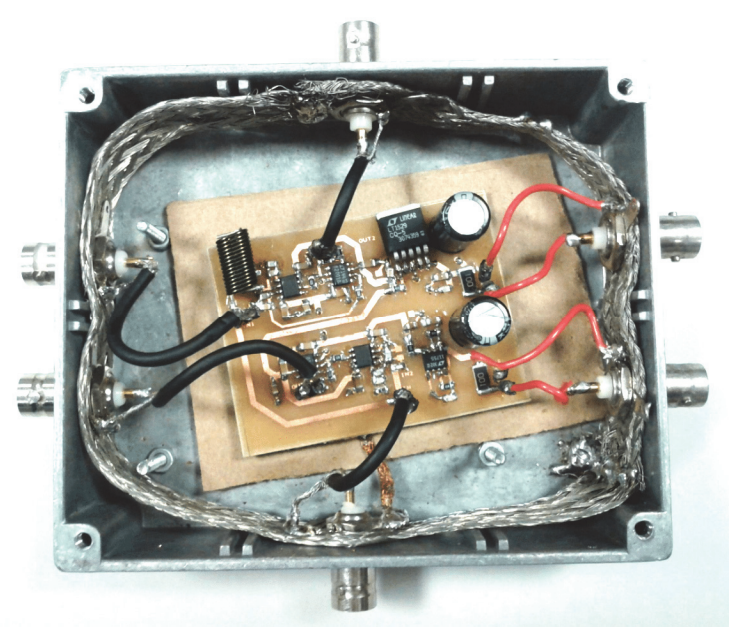

Fig. 4. Photograph of the test board with both schemes for comparison. 


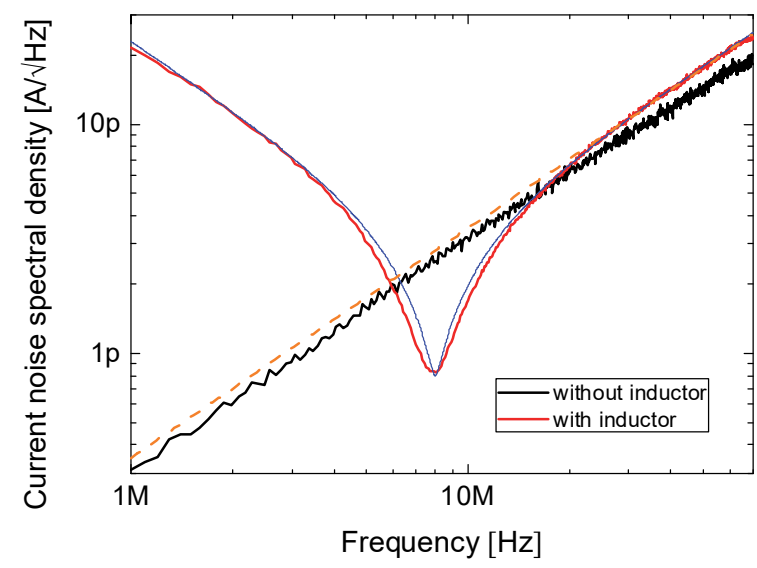

(a)

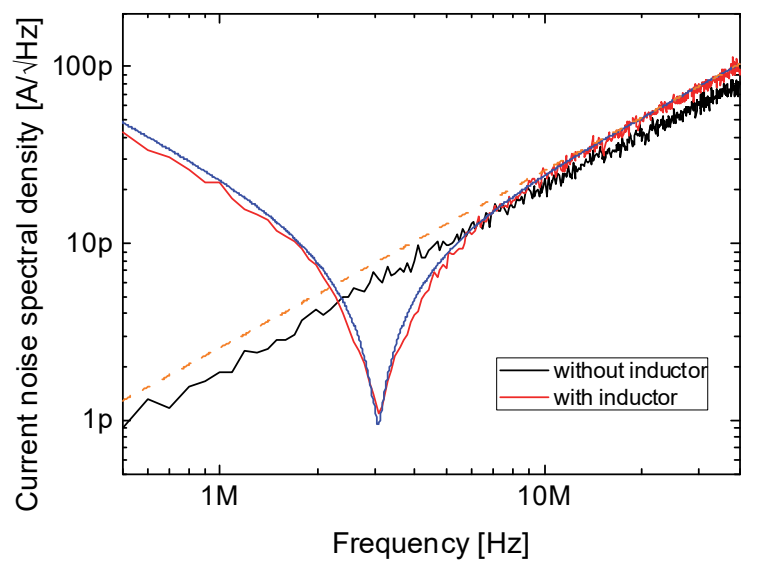

(b)

Fig. 5. Current noise spectral density from different versions of TIA implementing respectively $C_{\text {in }}=12 \mathrm{pF}$ (a) and $C_{i n}=100 \mathrm{pF}$ (b). The resonant amplifier uses an inductor of $L=22 \mu \mathrm{H}$. The red and black curves represents the measureed noise in TIA with the inductor (resonant topology) and without the inductor (standard TIA). The blue and the dashed orange curves are respectively the simulated noise accounting for the inductor losses and the simulated noise in a traditonal TIA.

$$
G_{\text {loop }, D C l o o p}(s) \approx \frac{K}{1+s R C} A(s)
$$

The stability of the DC loop is assured by choosing a negative gain $K$ and the pole of the low-pass filter lower than $f_{0} /\left(K A_{0}\right)$, where $f_{0}$ is the dominant pole of the operational amplifier.

Figures 3 and 4 show the circuit implemented to validate the new topology of transimpedance amplifier. We adopted a JFET operational amplifier (ADA4817 by Analog Devices) to reduce the equivalent input current noise at few $f A / \sqrt{H z}$, so we can consider this contribution negligible compared to its voltage noise. The large gain-bandwidth product $(410 \mathrm{MHz})$ of the ADA4817 allows the correct operation of the circuit for resonant frequencies of tens of $\mathrm{MHz}$. The gain stage $\mathrm{K}$ is implemented using the operational amplifier LT6200-10 (by Linear Technology) configured as an inverting amplifier with a gain of -10 and a bandwidth of $160 \mathrm{MHz}$. The output of the full circuit is taken at the output of this inverting amplifier to profit of its additional gain. The pole of the low pass filter is $0.5 \mathrm{~Hz}$ in order to operate the DC loop up to few $\mathrm{kHz}$, well below the dominant pole of the ADA4817 (about $230 \mathrm{kHz}$ ) and the resonance of the inductor $(>\mathrm{MHz})$.

The value of the inductor is chosen based on the input capacitance $C_{i n}$ of the experiment to have the resonant frequency in the range of $1-100 \mathrm{MHz}$. The lower limit is dictated by the low $\mathrm{Q}$ factor of large inductors $(>100 \mu \mathrm{H})$. On the other side, the resonance frequency must be in the circuit bandwidth. The last condition also poses a limitation to the minimum value of the feedback capacitor $C_{F}$.

\section{EXPERIMENTAL RESUlTS AND DisCUSSION}

The experimental validation of the proposed transimpedance amplifier has been carried out by comparing its noise performance and the noise of a traditional TIA. The latter was designed using the same operational amplifier (ADA4817) and the same capacitors $C_{F}$ and $C_{i n}$. The feedback resistor was selected to $R_{F}=1 \mathrm{G} \Omega$ to have a negligible thermal noise at the frequency of interest.

We have considered two different case studies with $C_{i n}=12 p F \quad\left(C_{F}=2 p F\right) \quad$ and $C_{i n}=100 p F \quad\left(C_{F}=12 p F\right)$, respectively, to evaluate the noise suppression in different conditions. In both cases we used a totally capacitive DUT and no stationary input current. The inductors used in the reported experiments $(22 \mu \mathrm{H}$ and $500 \mathrm{nH})$ were purchased by Coilcraft. We fabricated the circuit prototypes by adopting all SMD components (only exception is $L=500 \mathrm{nH}$ ). The prototypes were completed by a network to filter the supply voltage and placed in a closed metallic box acting as a Faraday cage. Noise spectrum measurements were performed by the Agilent $M X A$ $N 9020 A$ spectrum analyzer and the transfer functions were measured by the Agilent E5061B network analyzer.

The measured input-referred noise spectra in the case of $C_{i n}=12 \mathrm{pF}$ are plotted in Fig. 5a. The inductor added to the virtual ground is $L=22 \mu \mathrm{H}$. The noise of the standard TIA (black line) increases proportionally to the frequency in agreement with eq. (1) (orange line). The proposed TIA (red line) shows a dip in the noise spectrum in correspondence of the resonant frequency $f_{\text {res }}=9 \mathrm{MHz}$, as expected. The noise is suppressed by a factor $\approx 3.1$ in agreement with the eq. (3) (blue line) taking in account the losses of the inductor related to the quality factor $\left(\mathrm{Q} \approx 20\right.$ at $\left.f_{\text {res }}\right)$. The noise at frequencies below the resonance increases due to the decrease of the inductor impedance that produces a current noise of $\approx \bar{e}_{n}^{2} /(2 \pi f L)^{2}$. The 


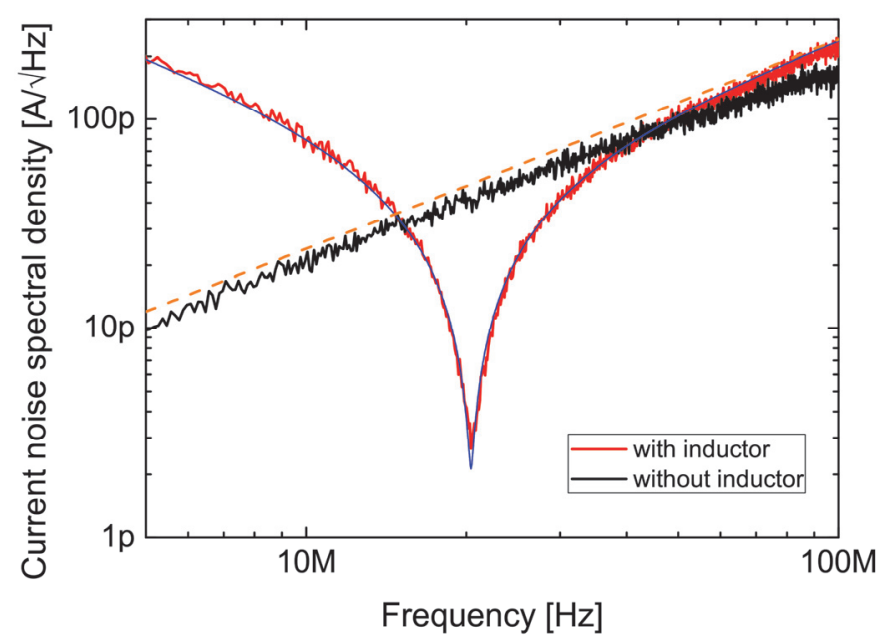

Fig. 6. Measured input-referred current noise for $C_{i n}=100 \mathrm{pF}$ with an inductor of $L=500 \mathrm{nH}$ (red line) and without the inductor (black line). The blue and the dashed orange curves are respectively the simulated noise with the inductor and the simulated noise in a traditonal TIA.

noise of the two circuits is very similar for frequencies higher than the resonance. The slight difference can be justified by gain mismatch between the fabricated amplifiers and to the additional input capacitance given by the inductor.

To quantify the improvement in the noise performance given by the proposed scheme, we define an improvement factor IF as the ratio of the noise in a standard TIA and the noise of the resonant circuit, both calculated at the frequency of the resonance:

$$
I F=\frac{\overline{i_{e q, T I A}^{2}}\left(f_{\text {res }}\right)}{\overline{i_{\text {eq, res }}^{2}}\left(f_{\text {res }}\right)}
$$

By assuming the noise of the TIA dominated by the voltage noise of the operational amplifier (see eq. (1)) and the noise of the resonant amplifier limited by the losses of the inductor (see eq. (3)), the improvement factor can be expressed as

$$
I F=\frac{\overline{e_{n}^{2}}}{4 k T} Q \sqrt{\frac{C_{i n}+C_{F}}{L}}
$$

In addition to the obvious observation on the importance of the quality factor of the resonance, eq. (7) suggests that the resonant scheme is particularly advantageous for large input capacitances. This trend is confirmed in the measurement reported in Fig. 5b. The noise spectra are obtained with an input capacitance of $C_{i n}=100 \mathrm{pF}$ and a feedback capacitance of $C_{F}=12 \mathrm{pF}$. In agreement with eq. (7), the noise suppression is doubled $(\approx 5.8)$ with respect to the case of $C_{i n}=12 \mathrm{pF}$. The improvement factor also profits of the higher quality factor of the inductor at the new resonant frequency $(\mathrm{Q} \approx 30$ at $3 \mathrm{MHz})$.

A high value of the input capacitor allows a further reduction of the noise by choosing a smaller inductor, as shown in Fig. 6 in the case of $C_{i n}=100 \mathrm{pF}$. The resonant amplifier is operated with an inductor of $\mathrm{L}=500 \mathrm{nH}$ obtaining a noise suppression of more than one order of magnitude $(\approx 14)$. The improvement has two reasons: i) the lower inductance

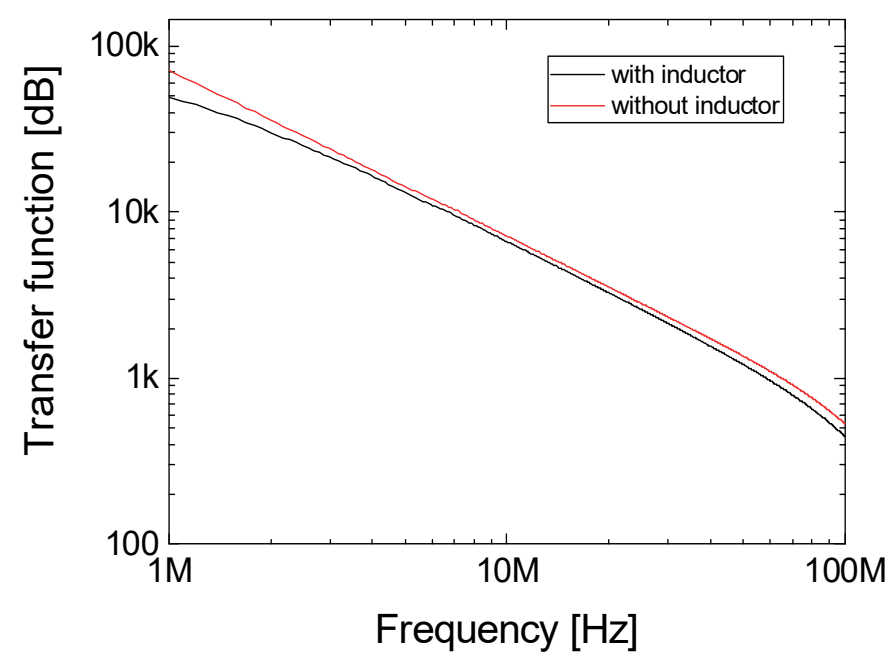

Fig. 7. Measured tranfer function $v_{\text {out }} / i_{\text {in }}$ of the resonant circuit (black line) with $L=500 \mathrm{nH}$ and $C_{i n}=100 \mathrm{pF}$ and of a standard TIA operated as an integrator $\left(C_{F}=12 \mathrm{pF}\right.$ and $R_{F}=1 G \Omega$, red line). The nominal values of $C_{i n}$ and $C_{F}$ are the same in the two circuits.

increases the resonant frequency and consequently the noise of the standard TIA at $f_{\text {res }}$ is increased; ii) a smaller inductance allows a higher quality factor, about 110 at $f_{\text {res }}$ in our case. Indeed, the improvement factor predicted by eq. (7) is a factor 2 higher than the measured one. This discrepancy is probably attributed to additional losses of the parasitic at the input node of the amplifier.

Finally, the frequency response of the resonant amplifier in the last configuration $\left(C_{i n}=100 \mathrm{pF}\right.$ and $\left.L=500 \mathrm{nH}\right)$ is shown in Fig. 7 together with the response of the standard TIA (operating as an integrator stage due to the large value of $R_{F}$ ) without the inductor at the input node. As expected, the gain of the amplifier is not affected at the resonance $\left(f_{\text {res }}=20 \mathrm{MHz}\right)$ by the presence of the inductor. Thus, the improvement of the signal-to-noise ratio is obtained without a reduction of the accuracy. The lower gain of the resonant amplifier at low frequency is due to the reduction of the loop gain determined by the small impedance of the inductor, as predicted by eq. (4).

\section{CONCLUSIONS}

We have demonstrated that high-sensitivity current measurements at a single frequency can be enabled by the addition of an inductor at the virtual ground node of a TIA. The inductive reactance nullifies the admittance at the virtual ground node of the amplifier and hampers the transfer to the output of the voltage noise of the operational amplifier. A simple additional network has been designed to keep a closedloop configuration of the operational amplifier at low frequency. We have experimentally demonstrated a noise suppression in excess of a factor 10 with respect to a traditional TIA stage. The improvement of noise occurs without alteration of the amplifier gain or a degradation of the accuracy at the resonant frequency, thus making the circuit ideal for highresolution impedance measurements at a single frequency. 


\section{ACKNOWLEDGMENT}

The authors would like to thank M. Sampietro for the many useful discussions on this topic. This work was supported by Politecnico di Milano under project Tid-Mekii and by the European Union through projects H2020-ICT-STREAMS (Grant No. 688172) and the Consolidator Grant VIBRA (ERC2014-CoG No. 648615).

\section{REFERENCES}

[1] M. Carminati, "Advances in high-resolution microscale impedance sensors," J. Sensors, vol. 2017, 7638389, pp. 1-15, 2017.

[2] A. Abdelkafi et al., "Investigation of the electrode surface of a liquid quality sensor by local impedance spectroscopy," 2014 IEEE International Instrumentation and Measurement Technology Conference (I2MTC) Proc., Montevideo, 2014, pp. 911-914.

[3] A. Sanli, A. Benchirouf, C. Müller, O. Kanoun, "Piezoresistive performance characterization of strain sensitive multi-walled carbon nanotube-epoxy nanocomposites," Sensors and Actuators A: Physical, vol. 254, pp. 61-68, 2017.

[4] P. Ciccarella, M. Carminati, M. Sampietro and G. Ferrari, "Multichannel $65 \mathrm{zF}$ rms resolution CMOS monolithic capacitive sensor for counting single micrometer-sized airborne particles on chip," IEEE J. Solid-State Circuits, vol. 51, no. 11, pp. 2545-2553, Nov. 2016.

[5] D. G. Muratore, E. Bonizzoni, F. Maloberti and C. Fiocchi, "A capacitive sensor interface for high-resolution acquisitions in hostile environments," 2016 IEEE 7th Latin American Symposium on Circuits \& Systems (LASCAS), Florianopolis, 2016, pp. 167-170.

[6] M. Carminati, G. Ferrari, M. D. Vahey, J. Voldman and M. Sampietro, "Miniaturized impedance flow cytometer: design rules and integrated readout," IEEE Trans. Biomedical Circuits and Systems, in press.

[7] M. Carminati et al., "Design guidelines for contactless integrated photonic probes in dense photonic circuits," J. Lightwave Technology, vol. 35, no. 14, pp. 3042-3049, July 2017.

[8] G. Gervasoni, M. Carminati, G. Ferrari, "Switched ratiometric lock-in amplifier enabling sub-ppm measurements in a wide frequency range," Rev. Sci. Instrum., vol. 88, 104704, 2017.

[9] J. K. Rosenstein, M. Wanunu, C. A. Merchant, M. Drndic, and K. L. Shepard, "Integrated nanopore sensing platform with sub-microsecond temporal resolution," Nature Methods, vol. 9, no. 5, pp. 487-492, 2012.

[10] M. Carminati, M. Vergani, G. Ferrari, L. Caranzi, M. Caironi, and M. Sampietro, "Accuracy and resolution limits in quartz and silicon substrates with microelectrodes for electrochemical biosensors," Sensors and Actuators, B: Chemical, vol. 174, pp. 168-175, 2012.

[11] M. Carminati, G. Ferrari, A.P. Ivanov, T. Albrecth, M. Sampietro, "Design and characterization of a current sensing platform for siliconbased nanopores with integrated tunneling nanoelectrodes," Analog Integr Circ Sig Process, vol. 77, pp. 333-343, 2013.

[12] R. Howard, "Ultralow noise high gain transimpedance amplifier for characterizing the low frequency noise of infrared detectors," Rev. Sci. Instrum., vol. 70, no. 3, pp. 1860-1867, 1999.

[13] G. Ferrari and M. Sampietro, "Wide bandwidth transimpedance amplifier for extremely high sensitivity continuous measurements," Rev. Sci. Instrum., vol. 78, no. 9, p. 94703, Sep. 2007

[14] J. Réhault, F. Crisafi, V. Kumar, G. Ciardi, M. Marangoni, G. Cerullo, D. Polli, "Broadband stimulated Raman scattering with Fouriertransform detection," Optics Express, vol. 23, no. 19, pp. 25235-25246, 2015.

[15] F. Crisafi, V. Kumar, T. Scopigno, M. Marangoni, G. Cerullo, D. Polli, "In-line balanced detection stimulated Raman scattering microscopy," Scientific Reports, vol. 7, no. 1, 10745, 2017.

[16] T. Tran, D. R. Oliver, D. J. Thomson, and G. E. Bridges, “'Zeptofarad' $(10-21 F)$ resolution capacitance sensor for scanning capacitance microscopy," Rev. Sci. Instrum., vol. 72, no. 6, pp. 2618-2623, 2001.

[17] L. Dicarlo, Y. Zhang, D. T. McClure, C. M. Marcus, L. N. Pfeiffer, and K. W. West, "System for measuring auto- And cross correlation of current noise at low temperatures," Rev. Sci. Instrum., vol. 77, no. 7, p. 73906, 2006.

[18] M. N. Slipchenko, R. A. Oglesbee, D. Zhang, W. Wu, and J. X. Cheng, "Heterodyne detected nonlinear optical imaging in a lock-in free manner," J. Biophotonics, vol. 5, no. 10, pp. 801-807, 2012.

[19] N. Haandbæk, O. With, S. C. Bürgel, F. Heer, and A. Hierlemann, "Resonance-enhanced microfluidic impedance cytometer for detection of single bacteria.," Lab Chip, pp. 3313-3324, 2014.

[20] C. Ghidini, D. Marioli, E. Sardini, and A. Taroni, “A 15 ppm resolution measurement system for capacitance transducers," Meas. Sci. Technol., vol. 7, no. 12, pp. 1787-1792, 1996. 